\title{
On the Paper: Numerical Radius Preserving Linear Maps on Banach Algebras
}

\author{
Mohammed El Azhari \\ Department of Mathematics, Ecole Normale Supérieure, Rabat, Morocco
}

Email address:

mohammed.elazhari@yahoo.fr

\section{To cite this article:}

Mohammed El Azhari. On the Paper: Numerical Radius Preserving Linear Maps on Banach Algebras. Mathematics Letters. Vol. 3, No. 5, 2017, pp. 50-51. doi: 10.11648/j.ml.20170305.11

Received: May 16, 2017; Accepted: September 8, 2017; Published: October 10, 2017

\begin{abstract}
We give an example of a unital commutative complex Banach algebra having a normalized state which is not a spectral state and admitting an extreme normalized state which is not multiplicative. This disproves two results by Golfarshchi and Khalilzadeh.
\end{abstract}

Keywords: Banach Algebra, Regular Norm, Normalized State, Spectral State

\section{Preliminaries}

Let $(A,\|\|$.$) be a complex normed algebra with an identity$ $e$ such that $\|e\|=1$. Let $D(A, e)=\left\{f \in A^{\prime}: f(e)=\|f\|=\right.$ $1\}$, where $A^{\prime}$ is the dual space of $A$. The elements of $D(A, e)$ are called normalized states on $A$. For $a \in A$, let $V(A, a)=$ $\{f(a): f \in D(A, e)\}, V(A, a)$ is called the numerical range of $a$. Let $\operatorname{sp}(a)$ be the spectrum of $a \in A$, and let $\operatorname{co}(\operatorname{sp}(a))$ be the convex hull of $\operatorname{sp}(a)$. We say that a linear functional $f$ on $A$ is a spectral state if $f(a) \in \operatorname{co}(\operatorname{sp}(a))$ for all $a \in A$. We denote by $M(A)$ the set of all non-zero continuous multiplicative linear functionals on $A$.

\section{Result}

\subsection{Counterexample}

Golfarshchi and Khalilzadeh proved the following results [4]:

[4, Theorem 2]. Let $A$ be a unital complex Banach algebra, and let $f$ be a linear functional on $A$. Then $f$ is a normalized state on $A$ if and only if $f(a) \in \operatorname{co}(\operatorname{sp}(a))$ for all $a \in A$.

[4, Theorem 3]. Let $A$ be a unital commutative complex Banach algebra. Then each extreme normalized state on $A$ is multiplicative.

Here we give a counterexample disproving the above results. We also remark that Theorems 5 and 6 [4] are called into question since the authors used Theorem 3 [4] to prove these results.

Let $(A,\|\|$.$) be a non-zero commutative radical complex$
Banach algebra [6, p.316]. Let $A_{e}=\{a+\lambda e: a \in A, \lambda \in \mathbb{C}\}$ be the unitization of $A$ with the identity $e$, and the norm $\|a+\lambda e\|_{1}=\|a\|+|\lambda|$ for all $a+\lambda e \in A_{e} \cdot\left(A_{e},\|.\|_{1}\right)$ is a unital commutative complex Banach algebra, and $M\left(A_{e}\right)=$ $\left\{\varphi_{\infty}\right\}$, where $\varphi_{\infty}$ is the continuous multiplicative linear functional on $A_{e}$ defined by $\varphi_{\infty}(a+\lambda e)=\lambda$ for all $a+\lambda e \in A_{e}$.

(1). let $a$ be a non-zero element of $A, V\left(A_{e}, a\right)=\{z \in$ $\mathbb{C}:|z| \leq\|a\|\}$ by $\left[2\right.$, Remark 3.8], and $\operatorname{sp}(a)=\left\{\varphi_{\infty}(a)\right\}=$ $\{0\}$, hence $\operatorname{co}(\operatorname{sp}(a))=\{0\}$ is strictly included in $V\left(A_{e}, a\right)$ since $\|a\| \neq 0$. Therefore the direct implication of $[4$, Theorem 2] does not hold.

(2). By [1, Lemma 1.10.3], $D\left(A_{e}, e\right)$ is a non-empty weak* compact convex subset of $A_{e}^{\prime}$, then $\operatorname{ext}\left(D\left(A_{e}, e\right)\right)$ is a nonempty set. Assume that each extreme normalized state on $A_{e}$ is multiplicative, then $\operatorname{ext}\left(D\left(A_{e}, e\right)\right)=\left\{\varphi_{\infty}\right\}$. Let $a$ be a non-zero element of $A$, by [1, Corollary 1.10 .15$]$ there exists $f \in D\left(A_{e}, e\right)$ such that $f(a) \neq 0=\varphi_{\infty}(a)$. Therefore $\overline{c o}\left(\operatorname{ext}\left(D\left(A_{e}, e\right)\right)\right)=\left\{\varphi_{\infty}\right\}$ is strictly included in $D\left(A_{e}, e\right)$, which contradicts the Krein-Milman Theorem. This shows that [4, Theorem 3] is not valid.

\subsection{Regular Norm and the Operator Seminorm}

Let $(A, \|$. I) $)$ be a non-unital complex Banach algebra, and let $A_{e}=\{a+\lambda e: a \in A, \lambda \in \mathbb{C}\}$ be the unitization of $A$ with the identity e. Let $\|a+\lambda e\|_{o p}=\sup \{\|(a+\lambda e) x\|, \| x(a+$ $\lambda e)\|: x \in A\|, x \| \leq 1\}$ for all $a+\lambda e \in A_{e},\|.\|_{o p}$ is an algebra seminorm on $A_{e}$. We say that $\|$.$\| is regular if$ $\|$. $\left\|_{o p}=\right\|$. $\|$ on $A$. If $\|$.$\| is regular, it is well known that$ $\left(A_{e},\left\|_{.}\right\|_{o p}\right)$ is a complex Banach algebra. The following 
question was asked [3]: If $\left(A_{e},\|.\|_{o p}\right)$ is a complex Banach algebra, is the norm II. II regular?

Orenstein tried to give an answer to this question in the commutative case [5], but his proof is not correct since it is essentially based on the direct implication of [4, Theorem 2].

\section{Conclusion}

In this note, we show that Theorems 2 and 3 [4] are false by giving a counterexample. We also remark that Theorems 5 and 6 [4] and Theorem 1.1 [5] are called into question since the authors used Theorems 2 or 3 [4] to prove these results.

\section{References}

[1] F. F. Bonsall and J. Duncan, Complete normed algebras, New York: Springer Verlag 1973.
[2] A. K. Gaur and T. Husain, Spatial numerical ranges of elements of Banach algebras, International Journal of Mathematics and Mathematical Sciences, 12(4)(1989), 633640 .

[3] A. K. Gaur and Z. V. Kováŕík, Norms, states and numerical ranges on direct sums, Analysis, 11(2-3)(1991), 155-164.

[4] F. Golfarshchi and A. A. Khalilzadeh, Numerical radius preserving linear maps on Banach algebras, International Journal of Pure and Applied Mathematics, 88(2)(2013), 233238 .

[5] A. Orenstein, Regular norm and the operator seminorm on a non-unital complex commutative Banach algebra, arXiv: 1410.8790v2 [math.FA] 11 Dec 2015.

[6] C. E. Rickart, General theory of Banach algebras, New York: Van Nostrand 1960. 